\title{
The Sonogashira Reaction on Quinolizium Cations
}

\author{
Domingo García, Ana M. Cuadro,* Julio Alvarez-Builla, \\ and Juan J. Vaquero* \\ Departamento de Química Orgánica, Universidad de Alcalá \\ 28871-Alcalá de Henares, Madrid. Spain \\ juanjose.vaquero@uah.es
}

Supporting Information

Experimental procedures and characterization data

for all new compounds reported

(8 pages )

\section{Experimental section}

General. Melting points were uncorrected. Infrared spectra were recorded on $\mathrm{KBr}$ or $\mathrm{NaCl}$ pellets and spectral bands were reported in $\mathrm{cm}^{-1} .{ }^{1} \mathrm{H}$ NMR spectra were recorded at 200 and 300 $\mathrm{MHz}$ and ${ }^{13} \mathrm{C}$ NMR at 50 and $75 \mathrm{MHz}$. Chemical shifts were reported as $\delta$ values (ppm). Lowresolution mass spectra (MS) were obtained as ESI (Na). CuI, $\mathrm{PdCl}_{2}\left(\mathrm{PPh}_{3}\right)_{2}$, were purchased from Aldrich. The acetylenes: triisopropylsilylacetylene, trimethylsilylacetylene, 1-ethynyl-4methyl-benzene, 1-ethynyl-4-methoxy-benzene, 1-ethynyl-4-trifluoromethyl-benzene and 2ethynylpyridine were purchased from Aldrich and were used without further purification. 1Bromo-3-ethynylbenzene was obtained by a previously described method. ${ }^{1}$ DMF, DMAC and $\mathrm{Et}_{3} \mathrm{~N}$ were distilled over activated molecular sieves.

Sonogashira Reactions on Quinolizinum Salts 5a-d. General Procedure for the Synthesis of Substituted Quinolizinium Cations 1-4.

Method A. A flame-dried vial was charged under argon with $50 \mathrm{mg}(0.173 \mathrm{mmol})$ of the corresponding bromoquinolizinium salt, $10 \mathrm{~mol} \% \mathrm{CuI}(3.3 \mathrm{mg}, 0.0173 \mathrm{mmol})$ and $5 \mathrm{~mol} \%$ $\mathrm{PdCl}_{2}\left(\mathrm{PPh}_{3}\right)_{2}(6.9 \mathrm{mg}, 0.0087 \mathrm{mmol})$ in dry DMF $(2.5 \mathrm{~mL})$. Then the corresponding acetylene $(0.208 \mathrm{mmol})$ and $\mathrm{Et}_{3} \mathrm{~N}(0.259 \mathrm{mmol}, 45 \mu \mathrm{L})$ were added. After heating at $60{ }^{\circ} \mathrm{C}$ for $14 \mathrm{~h}$, the solution was filtered through a small pad of celite and washed with methanol $(5 \mathrm{~mL})$. The solution was concentrated, treated with a saturated solution of $\mathrm{NaHCO}_{3}(20 \mathrm{~mL})$ and extracted with EtOAc $(15 \mathrm{~mL})$. The organic phase was extracted with $\mathrm{H}_{2} \mathrm{O}(2 \times 10 \mathrm{~mL})$, the aqueous

${ }^{1}$ Wettergren, J.; Minidis, A. B. E. Tetrahedron Lett. 2003, 16, 4467. 
phase was treated with a saturated solution of $\mathrm{NH}_{4} \mathrm{PF}_{6}$ and the resulting phase was extracted with $\mathrm{CH}_{2} \mathrm{Cl}_{2}(3 \times 15 \mathrm{~mL})$. The organic phase was dried over $\mathrm{Na}_{2} \mathrm{SO}_{4}$, the solvent evaporated under reduced pressure and the and the product isolated by column chromatography on silica gel using $\mathrm{CH}_{2} \mathrm{Cl}_{2} / \mathrm{MeOH}$ (9.6:0.4) as eluent.

Method B. A flame-dried vial was charged under argon with $50 \mathrm{mg}(0.173 \mathrm{mmol})$ of the corresponding bromoquinolizinium salt, $10 \mathrm{~mol} \% \mathrm{CuI}(3.3 \mathrm{mg}, 0.0173 \mathrm{mmol})$ and $5 \mathrm{~mol} \%$ $\mathrm{PdCl}_{2}\left(\mathrm{PPh}_{3}\right)_{2}(6.9 \mathrm{mg}, 0.0087 \mathrm{mmol})$ in dry $\mathrm{DMF}(2.5 \mathrm{~mL})$. Then 1.2 equiv.of the corresponding acetylene $(0.208 \mathrm{mmol})$ and 1.5 equiv. of $\mathrm{Et}_{3} \mathrm{~N}(0.259 \mathrm{mmol}, 45 \mu \mathrm{L})$ were added. After heating at $60{ }^{\circ} \mathrm{C}$ for $14 \mathrm{~h}$, the solution was filtered through a small pad of celite and washed with methanol $(5 \mathrm{~mL})$. The solution was concentrated, treated with $\mathrm{H}_{2} \mathrm{O}(20 \mathrm{~mL})$ and extracted with $\mathrm{CH}_{2} \mathrm{Cl}_{2}(3 \times 15 \mathrm{~mL})$. The organic phase was dried over $\mathrm{Na}_{2} \mathrm{SO}_{4}$, the solvent evaporated under reduced pressure and the and the product isolated by column chromatography on silica gel using $\mathrm{CH}_{2} \mathrm{Cl}_{2} / \mathrm{MeOH}$ (9.6:0.4) as eluent.

\section{2-(Triisopropylsilanylethynyl)quinolizinium bromide (2a).}

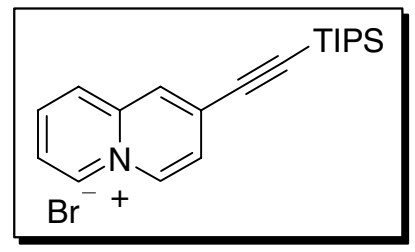

Following the general procedure $\mathrm{B}$, from $\mathbf{5 b}$ and triisopropylsilylacetylene $(47 \mu \mathrm{l})$, were obtained $55.6 \mathrm{mg}(82 \%)$ of 2a as a yellow solid: mp 206-208 ${ }^{\circ} \mathrm{C}\left(\mathrm{CH}_{2} \mathrm{Cl}_{2}: \mathrm{Et}_{2} \mathrm{O}\right)$; IR (NaCl) 2944, 1866, 2154, 1642, 1450, 1402, 1152, $883 \mathrm{~cm}^{-1} ;{ }^{1} \mathrm{H}$

RMN (200 MHz, Acetone- $\left.d_{6}\right): \delta 10.22(\mathrm{~d}, 1 \mathrm{H}, J=6.4 \mathrm{~Hz}), 10.19(\mathrm{~d}, 1 \mathrm{H}, J=7.2 \mathrm{~Hz}), 8.22(\mathrm{~s}$, $1 \mathrm{H}), 8.72(\mathrm{~d}, 1 \mathrm{H}, J=8.5 \mathrm{~Hz}), 8.51(\mathrm{ddd}, 1 \mathrm{H}, J=8.5,7.3,1.0 \mathrm{~Hz}$ ), 8.18 (ddd, $1 \mathrm{H}, J=8.2,6.9$, $1.3 \mathrm{~Hz}), 8.09$ (dd, $1 \mathrm{H}, J=7.2,1.8 \mathrm{~Hz}), 1.21-1.14(\mathrm{~m}, 21 \mathrm{H}) ;{ }^{13} \mathrm{C}$ RMN (50 MHz, Acetone- $\left.d_{6}\right): \delta$ $143.7,138.6,138.3,137.9,131.4,130.3,127.9,125.8,124.9,104.1,103.3,18.9,11.8$. MS $\left(\mathrm{ESI}^{+}\right) \mathrm{m} / \mathrm{z} 310\left(\mathrm{M}^{+}, 100\right)$. Anal. Calcd for $\mathrm{C}_{20} \mathrm{H}_{28}$ BrNSi: C, 61.53; H, 7.23; N, 3.59. Found: $\mathrm{C}$, $61.69 ; \mathrm{H}, 7.41 ; \mathrm{N}, 3.60$.

\section{2-(4-Methylophenyllethynyl)quinolizinium hexafluorophosphate (2b).}

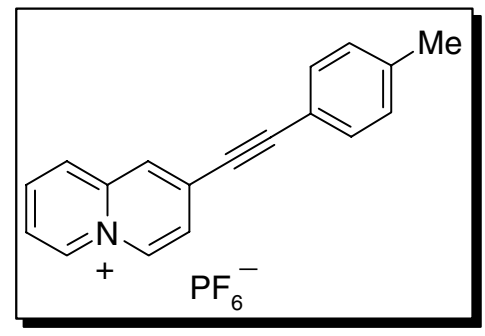

Following the general procedure A, from $\mathbf{5 b}$ and 1-ethynyl4-methyl-benzene (24.2 mg), were obtained $49.4 \mathrm{mg}$ (74\%) of $\mathbf{2 b}$ as a white solid: mp $205-207^{\circ} \mathrm{C}\left(\mathrm{CH}_{2} \mathrm{Cl}_{2}: \mathrm{Et}_{2} \mathrm{O}\right)$; IR (KBr) 2209, 1646, 1451, 1407, 1147, $837 \mathrm{~cm}^{-1}$; ${ }^{1} \mathrm{H}$ RMN $\left(300 \mathrm{MHz}\right.$, Acetone- $\left.d_{6}\right): \delta 9.42(\mathrm{~d}, 2 \mathrm{H}, J=7.0 \mathrm{~Hz}), 8.74(\mathrm{~s}$, 
$1 \mathrm{H}), 8.63(\mathrm{~d}, 1 \mathrm{H}, J=8.4 \mathrm{~Hz}), 8.51(\mathrm{t}, 1 \mathrm{H}, J=7.3 \mathrm{~Hz}), 8.21-8.19(\mathrm{~m}, 2 \mathrm{H}), 7.59(\mathrm{~d}, 2 \mathrm{H}, J=8.1$

$\mathrm{Hz}), 7.35(\mathrm{~d}, 2 \mathrm{H}, J=7.9 \mathrm{~Hz}), 2.40(\mathrm{~s}, 3 \mathrm{H}) ;{ }^{13} \mathrm{C} \mathrm{RMN}\left(75 \mathrm{MHz}\right.$, Acetone- $\left.d_{6}\right): \delta 143.5,141.8$, 138.3, 137.6, 137.3, 132.7, 132.2, 130.1, 129.1, 127.7, 125.7, 124.7, 118.2, 101.0, 85.7, 21.6. MS $\left(\mathrm{ESI}^{+}\right) \mathrm{m} / 2,244\left(\mathrm{M}^{+}, 100\right)$. Anal. Calcd for $\mathrm{C}_{18} \mathrm{H}_{14} \mathrm{~F}_{6} \mathrm{NP}: \mathrm{C}, 55.54 ; \mathrm{H}, 3.63 ; \mathrm{N}, 3.60$. Found: C, 55.60; H, 3.67; N, 3.39.

\section{2-(4-Methoxyphenylethynyl)quinolizinium hexafluorophosphate (2c).}

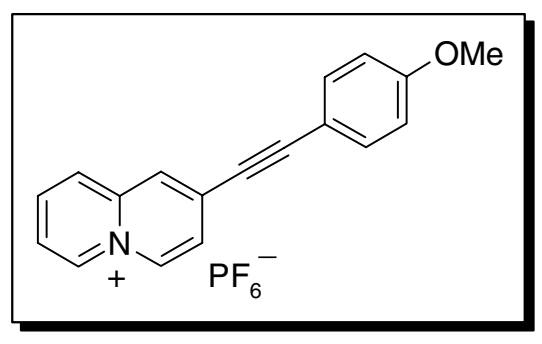

Following the general procedure $\mathrm{A}$, from $\mathbf{5 b}$ and 1ethynyl-4-methoxy-benzene $(27 \mu \mathrm{l})$, were obtained 54.9 mg (78\%) of 2c as a yellow solid: mp 195-196 ${ }^{\circ} \mathrm{C}$ $\left(\mathrm{CH}_{2} \mathrm{Cl}_{2}: \mathrm{Et}_{2} \mathrm{O}\right)$; IR (KBr) 3118, 2854, 2202, 1647, 1599, 1255, $842 \mathrm{~cm}^{-1} ;{ }^{1} \mathrm{H}$ RMN (300 MHz, Acetone- $\left.d_{6}\right): \delta$ $9.37(\mathrm{~d}, 2 \mathrm{H}, J=7.0 \mathrm{~Hz}), 8.70(\mathrm{~s}, 1 \mathrm{H}), 8.60(\mathrm{~d}, 1 \mathrm{H}, J=$ $8.4 \mathrm{~Hz}), 8.49$ (td, 1H, $J=7.9,0.9 \mathrm{~Hz}), 8.19-8.15(\mathrm{~m}, 2 \mathrm{H}), 7.66$ (d, 2H, $J=8.8 \mathrm{~Hz}), 7.07$ (d, 2H, $J=8.9 \mathrm{~Hz}), 3.89(\mathrm{~s}, 3 \mathrm{H}) ;{ }^{13} \mathrm{C} \mathrm{RMN}\left(75 \mathrm{MHz}\right.$, Acetone- $\left.d_{6}\right): \delta 162.7,144.0,138.6,138.0,137.6$, 135.1, 133.0, 129.0, 128.0, 126.0, 124.9, 115.5, 113.4, 102.0, 85.8, 55.9. MS (ESI $\left.{ }^{+}\right) \mathrm{m} / z 260$ $\left(\mathrm{M}^{+}, 100\right)$. Anal. Calcd for $\mathrm{C}_{18} \mathrm{H}_{14} \mathrm{~F}_{6} \mathrm{NOP}: \mathrm{C}, 53.35 ; \mathrm{H}, 3.48$; N, 3.46. Found: $\mathrm{C}, 53.46 ; \mathrm{H}, 3.57$; $\mathrm{N}, 3.68$.

\section{2-(4-Trifluoromethylphenylethynyl)-quinolizinium hexafluorophosphate (2d).}

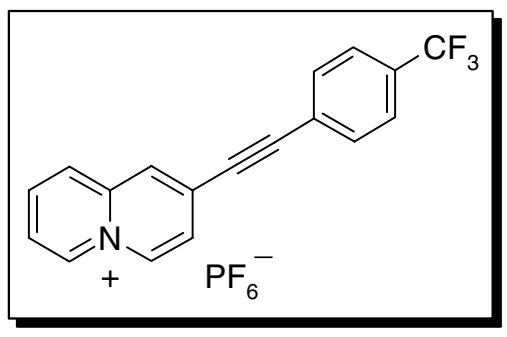

Following the general procedure A, from $\mathbf{5 b}$ and 1-ethynyl4-trifluoromethyl-benzene $(34 \mu \mathrm{l})$, were obtained $52.6 \mathrm{mg}$ $(69 \%)$ of $\mathbf{2 d}$ as a white solid: $\mathrm{mp}$ 213-214 ${ }^{\circ} \mathrm{C}$ $\left(\mathrm{CH}_{2} \mathrm{Cl}_{2}: \mathrm{Et}_{2} \mathrm{O}\right)$; IR (KBr) 3109, 2226, 1643, 1409, 1327, 1132, 1067, $838 \mathrm{~cm}^{-1} ;{ }^{1} \mathrm{H}$ RMN (300 MHz, Acetone- $\left.d_{6}\right): \delta$ $9.44(\mathrm{~d}, 2 \mathrm{H}, J=7.0 \mathrm{~Hz}), 8.85(\mathrm{~s}, 1 \mathrm{H}), 8.66(\mathrm{~d}, 1 \mathrm{H}, J=8.6$ $\mathrm{Hz}), 8.56(\mathrm{td}, 1 \mathrm{H}, J=7.1,0.9 \mathrm{~Hz}), 8.28-8.22(\mathrm{~m}, 2 \mathrm{H}), 7.94(\mathrm{~d}, 2 \mathrm{H}, J=8.2 \mathrm{~Hz}), 7.87(\mathrm{~d}, 2 \mathrm{H}, J=$ $8.2 \mathrm{~Hz}$ ); ${ }^{13} \mathrm{C}$ RMN (75 MHz, Acetone- $\left.d_{6}\right): \delta 144.0,139.1,138.4,137.9,133.8,132.2$ (c, $J=32.3$ Hz), 131.6, 130.5, 128.4, 126.7 (c, $J=2.9 \mathrm{~Hz}), 126.1,125.8,125.6,124.7$ (c, $J=253.7 \mathrm{~Hz}$ ), 98.3, 88.0. MS $\left(\mathrm{ESI}^{+}\right) \mathrm{m} / z 298\left(\mathrm{M}^{+}, 100\right)$. Anal. Calcd for $\mathrm{C}_{18} \mathrm{H}_{11} \mathrm{~F}_{9} \mathrm{NP}: \mathrm{C}, 48.78 ; \mathrm{H}, 2.50 ; \mathrm{N}$, 3.16. Found: C, 48.89; H, 2.31; N, 3.25. 
2-(3-Bromophenylethynyl)quinolizinium hexafluorophosphate (2e).

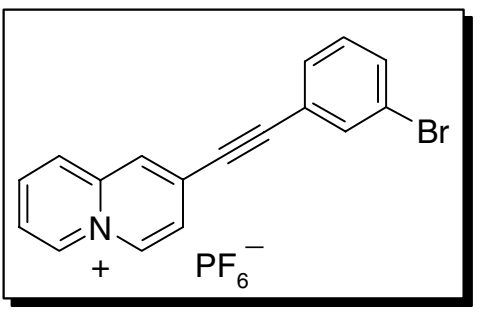

Following the general procedure A, from $\mathbf{5 b}$ and 1-bromo3-ethynyl-benzene (37.6 mg), were obtained $55.7 \mathrm{mg}$ (71\%) of $\mathbf{2 e}$ as a white solid: $\mathrm{mp} \quad 220-222{ }^{\circ} \mathrm{C}$ $\left(\mathrm{CH}_{2} \mathrm{Cl}_{2}: \mathrm{Et}_{2} \mathrm{O}\right)$; IR (KBr) 3111, 2234, 2218, 1643, 1451, 1409, 1151, $844 \mathrm{~cm}^{-1} ;{ }^{1} \mathrm{H}$ RMN (200 MHz, Acetone- $\left.d_{6}\right): \delta$

$9.41(\mathrm{~d}, 2 \mathrm{H}, J=7.2 \mathrm{~Hz}), 8.79$ (s, $1 \mathrm{H}), 8.64(\mathrm{~d}, 1 \mathrm{H}, J=8.2 \mathrm{~Hz}), 8.53$ (td, $1 \mathrm{H}, J=8.0,0.8 \mathrm{~Hz}$ ), 8.25-8.18 (m, 2H), $7.88(\mathrm{t}, 1 \mathrm{H}, J=1.8 \mathrm{~Hz}), 7.77-7.69(\mathrm{~m}, 2 \mathrm{H}), 7.48(\mathrm{t}, 1 \mathrm{H}, J=7.7 \mathrm{~Hz}) ;{ }^{13} \mathrm{C}$ RMN (50 MHz, Acetone- $\left.d_{6}\right): \delta 143.9,139.0,138.2,137.8,135.5,134.5,132.0,131.8,130.2$, 128.3, 126.1, 125.4, 123.9, 122.9, 98.5, 87.2. MS (ESI $\left.{ }^{+}\right) m / z 310\left(\mathrm{M}^{+}+1,98\right), 308\left(\mathrm{M}^{+}-1,100\right)$. Anal. Calcd for $\mathrm{C}_{17} \mathrm{H}_{11} \mathrm{BrF}_{6} \mathrm{NP}$ : C, 44.96; H, 2.44; N, 3.08. Found: C, 44.80; H, 2.35; N, 3.33.

\section{2-(Pyridin-2-ylethynyl)quinolizinium hexafluorophosphate (2f).}

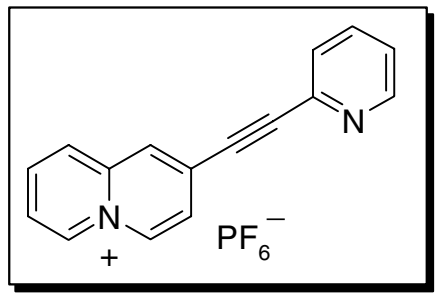

Following the general procedure A, from $\mathbf{5 b}$ and 2-ethynylpyridine $(21.4 \mathrm{mg}$ ), were obtained $28.2 \mathrm{mg}(43 \%)$ of $\mathbf{2 f}$ as a white solid: mp 219-222 ${ }^{\circ} \mathrm{C}\left(\mathrm{CH}_{2} \mathrm{Cl}_{2}: \mathrm{Et}_{2} \mathrm{O}\right)$; IR (KBr) 3111, 2213, 1643, 1618, 1449, 1408, $831 \mathrm{~cm}^{-1}$; ${ }^{1} \mathrm{H}$ RMN (300 MHz, Acetone- $\left.d_{6}\right): \delta 9.44(\mathrm{~d}, 2 \mathrm{H}, J=7.1 \mathrm{~Hz}), 8.86(\mathrm{~s}, 1 \mathrm{H}), 8.72(\mathrm{~d}$, $1 \mathrm{H}, J=4.8 \mathrm{~Hz}), 8.67(\mathrm{~d}, 1 \mathrm{H}, J=8.6 \mathrm{~Hz}), 8.56$ (ddd, $1 \mathrm{H}, J=$ 8.6, 8.3, $1.1 \mathrm{~Hz}), 8.28-8.22(\mathrm{~m}, 2 \mathrm{H}), 7.95(\mathrm{td}, 1 \mathrm{H}, J=7.7,1.7 \mathrm{~Hz}), 7.80(\mathrm{dt}, 1 \mathrm{H}, J=7.7,1.1$ $\mathrm{Hz}), 7.53(\mathrm{ddd}, 1 \mathrm{H}, J=7.7,6.0,1.1 \mathrm{~Hz}) ;{ }^{13} \mathrm{C} \mathrm{RMN}\left(75 \mathrm{MHz}\right.$, Acetone- $\left.d_{6}\right): \delta 151.3,143.7$, 141.9, 138.9, 138.1, 137.7, 137.5, 131.3, 130.5, 129.1, 128.1, 125.9, 125.5, 125.4, 98.8, 84.1. MS $\left(\operatorname{ESI}^{+}\right) \mathrm{m} / z 231\left(\mathrm{M}^{+}, 100\right)$. Anal. Calcd for $\mathrm{C}_{16} \mathrm{H}_{11} \mathrm{~F}_{6} \mathrm{~N}_{2} \mathrm{P}: \mathrm{C}, 51.08 ; \mathrm{H}, 2.95 ; \mathrm{N}, 7.45$. Found: C, 51.32; H, 2.71; N, 7.70.

\section{3-(Triisopropylsilanylethynyl)quinolizinium hexafluorophosphate (3a).}

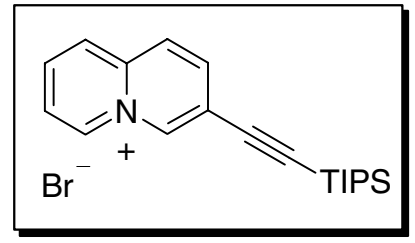

Following the general procedure $\mathrm{B}$, from $\mathbf{5 c}$ and triisopropylsilylacetylene $(47 \mu \mathrm{l})$, were obtained $54.2 \mathrm{mg}(80 \%)$ of 3a as a yellow solid: mp $70-72{ }^{\circ} \mathrm{C}\left(\mathrm{CH}_{2} \mathrm{Cl}_{2}: \mathrm{Et}_{2} \mathrm{O}\right)$; IR $(\mathrm{NaCl})$ 2944, 2865, 2163, 1626, 1463, 1398, 1222, 996, $882 \mathrm{~cm}^{-1} ;{ }^{1} \mathrm{H}$

RMN (300 MHz, Acetone- $\left.d_{6}\right): \delta 10.03(\mathrm{~s}, 1 \mathrm{H}), 9.81(\mathrm{~d}, 1 \mathrm{H}, J=6.6 \mathrm{~Hz}), 8.76(\mathrm{t}, 2 \mathrm{H}, J=9.5$ $\mathrm{Hz}), 8.55$ (ddd, 1H, $J=8.5,7.3,1.1 \mathrm{~Hz}), 8.45(\mathrm{dd}, 1 \mathrm{H}, J=9.0,1.7 \mathrm{~Hz}), 8.26(\mathrm{t}, 1 \mathrm{H}, J=6.5 \mathrm{~Hz}$ ), 1.21-1.15 (m, 21H); ${ }^{13} \mathrm{C}$ RMN (75 MHz, Acetone- $\left.d_{6}\right): \delta 143.0,140.2,138.9,138.6,137.7$, 
128.2, 128.1, 125.2, 120.2, 100.8, 100.4, 18.9, 11.8. $\mathrm{MS}\left(\mathrm{ESI}^{+}\right) \mathrm{m} / z 310\left(\mathrm{M}^{+}, 100\right)$. Anal. Calcd for $\mathrm{C}_{20} \mathrm{H}_{28} \mathrm{BrNSi}$ : C, 61.53; H, 7.23; N, 3.59. Found: C, 61.42; H, 7.51; N, 3.43.

\section{3-p-Tolylethynyl-quinolizinium hexafluorophosphate (3b).}

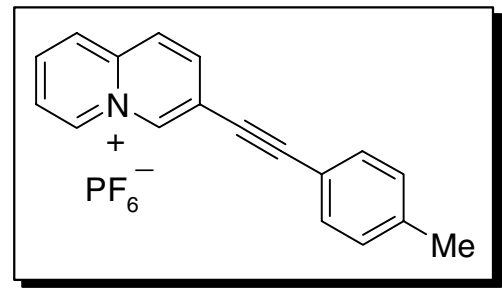

Following the general procedure $\mathrm{A}$, from $\mathbf{5 c}$ and 1ethynyl-4-methyl-benzene $(24.2 \mathrm{mg})$, were obtained 51.0 $\mathrm{mg}(76 \%)$ of $\mathbf{3 b}$ as a white solid: $\mathrm{mp} 207-209{ }^{\circ} \mathrm{C}$ $\left(\mathrm{CH}_{2} \mathrm{Cl}_{2}: \mathrm{Et}_{2} \mathrm{O}\right)$; IR (KBr) 2924, 2219, 1627, 1517, 1401, $831 \mathrm{~cm}^{-1} ;{ }^{1} \mathrm{H}$ RMN (200 MHz, Acetone- $\left.d_{6}\right): \delta 9.67(\mathrm{~s}$, $1 \mathrm{H}), 9.41(\mathrm{~d}, 1 \mathrm{H}, J=6.7 \mathrm{~Hz}), 8.71-8.66(\mathrm{~m}, 2 \mathrm{H}), 8.53(\mathrm{ddd}, 2 \mathrm{H}, J=9.5,7.9,1.0 \mathrm{~Hz}), 8.28(\mathrm{t}$, $1 \mathrm{H}, J=6.9 \mathrm{~Hz}), 7.55(\mathrm{~d}, 2 \mathrm{H}, J=8.2 \mathrm{~Hz}), 7.32(\mathrm{~d}, 2 \mathrm{H}, J=8.0 \mathrm{~Hz}), 2.40(\mathrm{~s}, 3 \mathrm{H}) ;{ }^{13} \mathrm{C} \mathrm{RMN}(75$ MHz, Acetone- $\left.d_{6}\right): \delta 142.9,141.6,139.4,139.1,138.6,137.5,132.8,130.4,128.3,128.2,125.8$, 121.5, 118.8, 97.9, 83.1, 21.5. MS (ESI $\left.{ }^{+}\right) m / z 244\left(\mathrm{M}^{+}, 100\right)$. Anal. Calcd for $\mathrm{C}_{18} \mathrm{H}_{14} \mathrm{~F}_{6} \mathrm{NP}: \mathrm{C}$, $55.54 ; \mathrm{H}, 3.63 ; \mathrm{N}, 3.60$. Found: C, 55.25; H, 3.84; N, 3.54 .

\section{3-(4-Methoxyphenylethynyl)quinolizinium hexafluorophosphate (3c).}

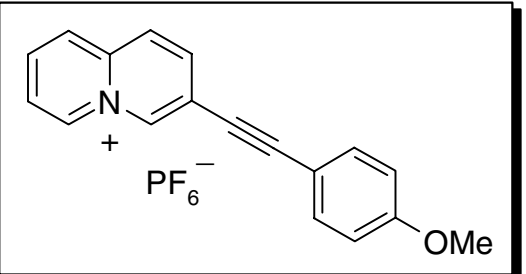

Following the general procedure A, from $\mathbf{5 c}$ and 1ethynyl-4-methoxy-benzene $(27 \mu \mathrm{l})$, were obtained $54.2 \mathrm{mg}(77 \%)$ of $\mathbf{3 c}$ as a yellow solid: $\mathrm{mp} 197-198^{\circ} \mathrm{C}$ $\left(\mathrm{CH}_{2} \mathrm{Cl}_{2}: \mathrm{Et}_{2} \mathrm{O}\right)$; IR (KBr) 3106, 2853, 2210, 1716, 1629, 1599, 1516, 1254, $842 \mathrm{~cm}^{-1}$; ${ }^{1} \mathrm{H}$ RMN $(300 \mathrm{MHz}$, Acetone- $\left.d_{6}\right): \delta 9.64(\mathrm{~s}, 1 \mathrm{H}), 9.41(\mathrm{~d}, 1 \mathrm{H}, J=6.8 \mathrm{~Hz}), 8.70(\mathrm{~d}, 1 \mathrm{H}, J=5.3 \mathrm{~Hz}), 8.67(\mathrm{~d}, 1 \mathrm{H}, J=$ $5.3 \mathrm{~Hz}) 8.56-8.48(\mathrm{~m}, 2 \mathrm{H}), 8.27(\mathrm{t}, 1 \mathrm{H}, J=7.0 \mathrm{~Hz}), 7.61(\mathrm{~d}, 2 \mathrm{H}, J=9.0 \mathrm{~Hz}), 7.06(\mathrm{~d}, 2 \mathrm{H}, J=$ $9.0 \mathrm{~Hz}), 3.88(\mathrm{~s}, 3 \mathrm{H}) ;{ }^{13} \mathrm{C} \mathrm{RMN}\left(75 \mathrm{MHz}\right.$, Acetone- $\left.d_{6}\right): \delta$ 162.2, 142.8, 139.1, 138.4, 137.5, 134.6, 128.3, 128.2, 125.7, 121.9, 115.4, 113.6, 98.2, 94.0, 82.6, 55.9. MS $\left(\mathrm{ESI}^{+}\right) \mathrm{m} / z 260\left(\mathrm{M}^{+}\right.$, 100). Anal. Calcd for $\mathrm{C}_{18} \mathrm{H}_{14} \mathrm{~F}_{6} \mathrm{NOP}: \mathrm{C}, 55.35 ; \mathrm{H}, 3.48 ; \mathrm{N}, 3.46$. Found: $\mathrm{C}, 55.12 ; \mathrm{H}, 3.63 ; \mathrm{N}$, 3.19 .

\section{3-(4-Trifluoromethylphenylethynyl)quinolizinium hexafluorophosphate (3d).}

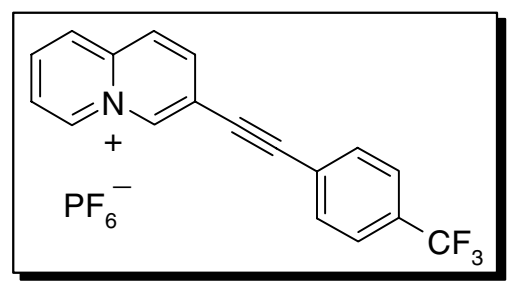

Following the general procedure A, from $\mathbf{5 c}$ and 1ethynyl-4-trifluoromethyl-benzene $(34 \mu 1)$, were obtained $44.6 \mathrm{mg}(58 \%)$ of $\mathbf{3 d}$ as a white solid: $\mathrm{mp} 202-204{ }^{\circ} \mathrm{C}$ $\left(\mathrm{CH}_{2} \mathrm{Cl}_{2}: \mathrm{Et}_{2} \mathrm{O}\right)$; IR (KBr) 3121, 2223, 1629, 1404, 1327, 
1130, 1067, $839 \mathrm{~cm}^{-1} ;{ }^{1} \mathrm{H}$ RMN (300 MHz, Acetone- $\left.d_{6}\right): \delta 9.74(\mathrm{~s}, 1 \mathrm{H}), 9.41(\mathrm{~d}, 1 \mathrm{H}, J=6.8$

$\mathrm{Hz}), 8.71(\mathrm{~d}, 2 \mathrm{H}, J=9.0 \mathrm{~Hz}), 8.59-8.54(\mathrm{~m}, 2 \mathrm{H}), 8.29(\mathrm{t}, 1 \mathrm{H}, J=7.0 \mathrm{~Hz}), 7.91(\mathrm{~d}, 2 \mathrm{H}, J=8.2$

$\mathrm{Hz}), 7.85(\mathrm{~d}, 2 \mathrm{H}, J=8.4 \mathrm{~Hz}) ;{ }^{13} \mathrm{C} \mathrm{RMN}\left(75 \mathrm{MHz}\right.$, Acetone- $\left.d_{6}\right): \delta 143.3,140.1,139.1,139.0$, 137.8, 133.5, 132.8 (c, $J=32.3 \mathrm{~Hz}), 128.4,126.7$ (c, $J=4.4 \mathrm{~Hz}), 126.0,125.9,124.8$ (c, $J=$ 256.6 Hz), 122.9, 120.6, 95.3, 85.6. MS (ESI $\left.{ }^{+}\right) \mathrm{m} / z 298\left(\mathrm{M}^{+}, 100\right)$. Anal. Calcd for $\mathrm{C}_{18} \mathrm{H}_{11} \mathrm{~F}_{9} \mathrm{NP}$ :

C, 48.78; H, 2.50; N, 3.16. Found: C, 48.53; H, 2.69; N, 3.15 .

\section{3-(3-Bromophenylethynyl)quinolizinium hexafluorophosphate (3e).}

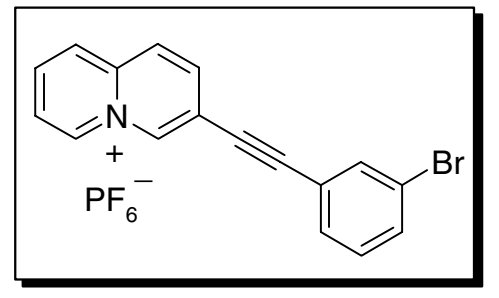

Following the general procedure A, from $\mathbf{5 c}$ and 1-bromo3-ethynyl-benzene $(37.6 \mathrm{mg})$, were obtained $54.9 \mathrm{mg}(70 \%)$ of 3e as a white solid: mp $224-226{ }^{\circ} \mathrm{C}\left(\mathrm{CH}_{2} \mathrm{Cl}_{2}: \mathrm{Et}_{2} \mathrm{O}\right)$; IR (KBr) 3110, 2230, 1627, 1511, 1399, $844 \mathrm{~cm}^{-1} ;{ }^{1} \mathrm{H}$ RMN (300 MHz, Acetone- $\left.d_{6}\right): \delta 9.71(\mathrm{~s}, 1 \mathrm{H}), 9.41(\mathrm{~d}, 1 \mathrm{H}, J=6.8$

$\mathrm{Hz}), 8.71(\mathrm{~d}, 2 \mathrm{H}, J=9.0 \mathrm{~Hz}), 8.56(\mathrm{td}, 2 \mathrm{H}, J=8.4,1.1 \mathrm{~Hz}), 8.29(\mathrm{t}, 1 \mathrm{H}, J=6.2 \mathrm{~Hz}), 7.85(\mathrm{t}, 1 \mathrm{H}$, $J=1.5 \mathrm{~Hz}), 7.73(\mathrm{ddd}, 1 \mathrm{H}, J=8.1,1.8,0.9 \mathrm{~Hz}), 7.68(\mathrm{dt}, 1 \mathrm{H}, J=7.9,1.3 \mathrm{~Hz}), 7.48(\mathrm{t}, 1 \mathrm{H}, J=$ 8.1, Hz); ${ }^{13} \mathrm{C}$ RMN (75 MHz, Acetone- $\left.d_{6}\right): \delta$ 143.0, 139.7, 138.8, 138.7, 137.5, 135.0, 133.9, 131.5, 131.4, 128.1, 125.7, 123.8, 122.7, 120.5, 95.1, 84.6. MS (ESI $\left.{ }^{+}\right) \mathrm{m} / z 310\left(\mathrm{M}^{+}+1,99\right), 308$ $\left(\mathrm{M}^{+}-1,100\right)$. Anal. Calcd for $\mathrm{C}_{17} \mathrm{H}_{11} \mathrm{BrF}_{6} \mathrm{NP}: \mathrm{C}, 44.96$; H, 2.44; N, 3.08. Found: $\mathrm{C}, 44.72 ; \mathrm{H}$, $2.73 ; \mathrm{N}, 3.03$.

\section{3-(Pyridin-2-ylethynyl)quinolizinium hexafluorophosphate (3f).}

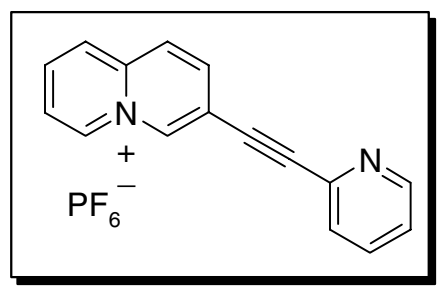

Following the general procedure A, from $\mathbf{5 c}$ and 2-ethynylpyridine $(21.4 \mathrm{mg}$ ), were obtained $32.7 \mathrm{mg}(50 \%)$ of $\mathbf{3 f}$ as a light brown solid: $\mathrm{mp} 220-222{ }^{\circ} \mathrm{C}\left(\right.$ Acetone: $\left._{2} \mathrm{O}\right)$; IR $(\mathrm{KBr})$ 2925, 1628, 1431, 1386, $1841 \mathrm{~cm}^{-1}$; ${ }^{1} \mathrm{H}$ RMN (300 MHz, Acetone- $\left.d_{6}\right): \delta 9.77(\mathrm{~s}, 1 \mathrm{H}), 9.45(\mathrm{~d}, 1 \mathrm{H}, J=6.8 \mathrm{~Hz}), 8.74-8.72$ (m, 3H), 8.61-8.55 (m, 2H), $8.31(\mathrm{t}, 1 \mathrm{H}, J=7.1 \mathrm{~Hz}), 7.94(\mathrm{td}, 1 \mathrm{H}, J=7.8,1.7 \mathrm{~Hz}), 7.76(\mathrm{dt}, 1 \mathrm{H}$, $J=7.9,0.9 \mathrm{~Hz}), 7.52(\mathrm{ddd}, 1 \mathrm{H}, J=7.7,6.0,1.3 \mathrm{~Hz}) ;{ }^{13} \mathrm{C} \mathrm{RMN}\left(75 \mathrm{MHz}\right.$, Acetone- $\left.d_{6}\right): \delta 151.3$, 143.2, 142.1, 140.3, 139.0, 138.9, 137.7, 137.5, 128.9, 128.3, 125.8, 125.3, 120.4, 96.1, 81.9. MS $\left(\mathrm{ESI}^{+}\right) \mathrm{m} / z 231\left(\mathrm{M}^{+}, 100\right)$. Anal. Calcd for $\mathrm{C}_{16} \mathrm{H}_{11} \mathrm{~F}_{6} \mathrm{~N}_{2} \mathrm{P}: \mathrm{C}, 51.08 ; \mathrm{H}, 2.95 ; \mathrm{N}, 7.45$. Found: C, 49.92; H, 3.09; N, 7.36. 
1-(4-Methoxy-phenylethynyl)-quinolizinium hexafluorophosphate (1a).

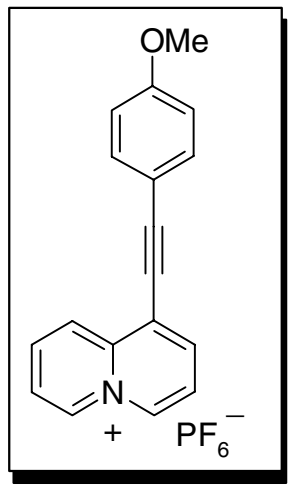

Following the general procedure A, from 5a and 1-ethynyl-4-methoxybenzene $(27 \mu \mathrm{l})$, were obtained $33.1 \mathrm{mg}(47 \%)$ of $\mathbf{1 a}$ as a yellow solid: mp 235-237 ${ }^{\circ} \mathrm{C}$; IR (KBr) 3123, 2205, 1638, 1606, 1257, 837, $557 \mathrm{~cm}^{-1}$; ${ }^{1} \mathrm{H}$ RMN (300 MHz, Acetone- $\left.d_{6}\right): \delta 9.51(\mathrm{~d}, 1 \mathrm{H}, J=6.8 \mathrm{~Hz}), 9.39(\mathrm{~d}, 1 \mathrm{H}$, $J=7.0 \mathrm{~Hz}), 9.11(\mathrm{~d}, 1 \mathrm{H}, J=8.6 \mathrm{~Hz}), 8.66-8.61(\mathrm{~m}, 2 \mathrm{H}), 8.29(\mathrm{td}, 1 \mathrm{H}, J$ $=6.8,1.3 \mathrm{~Hz}), 8.21(\mathrm{t}, 1 \mathrm{H}, J=7.2 \mathrm{~Hz}), 7.74(\mathrm{~d}, 2 \mathrm{H}, J=9.0 \mathrm{~Hz}), 7.08(\mathrm{~d}$, $2 \mathrm{H}, J=9.0 \mathrm{~Hz}), 3.89(\mathrm{~s}, 3 \mathrm{H}) ;{ }^{13} \mathrm{C} \mathrm{RMN}\left(75 \mathrm{MHz}\right.$, Acetone- $\left.d_{6}\right): \delta 162.4$, $144.2,139.9,139.6,138.9,137.0,134.8,126.8,125.7,124.5,123.1$, 115.5, 113.6, 102.2, 81.6, 55.9. MS (ESI $\left.{ }^{+}\right) \mathrm{m} / \mathrm{z} 260\left(\mathrm{M}^{+}, 100\right)$. Anal. Calcd for $\mathrm{C}_{18} \mathrm{H}_{14} \mathrm{~F}_{6} \mathrm{NOP}: \mathrm{C}$, 53.53 ; H, 3.48; N, 3.46. Found: C, 53.40; H, 3.56; N, 3.71.

\section{1-(4-Trifluoromethyl-phenylethynyl)-quinolizinium hexafluorophosphate (1b).}

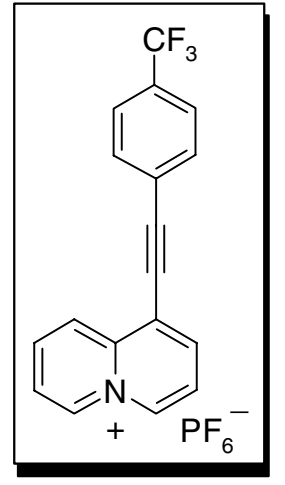

Following the general procedure A, from 5a and 1-ethynyl-4trifluoromethylbenzene $(34 \mu \mathrm{l})$, afforded $30.7 \mathrm{mg}(40 \%)$ of $\mathbf{1 b}$ as a white solid: mp 197-199 ${ }^{\circ} \mathrm{C}$; IR (KBr) 3121, 2926, 2224, 1636, 1410, 1326, 1133, 834, $558 \mathrm{~cm}^{-1} ;{ }^{1} \mathrm{H}$ RMN (300 MHz, Acetone- $\left.d_{6}\right): \delta 9.57(\mathrm{~d}, 1 \mathrm{H}, J=$ $6.8 \mathrm{~Hz}), 9.49(\mathrm{~d}, 1 \mathrm{H}, J=7.0 \mathrm{~Hz}), 9.18(\mathrm{~d}, 1 \mathrm{H}, J=8.6 \mathrm{~Hz}), 8.76$ (d, 1H, $J=$ $7.5 \mathrm{~Hz}), 8.68(\mathrm{td}, 1 \mathrm{H}, J=7.5,1.1 \mathrm{~Hz}), 8.35(\mathrm{t}, 1 \mathrm{H}, J=6.0 \mathrm{~Hz}), 8.27 \quad(\mathrm{t}$, $1 \mathrm{H}, J=7.1 \mathrm{~Hz}), 8.04(\mathrm{~d}, 2 \mathrm{H}, J=8.1 \mathrm{~Hz}), 7.89(\mathrm{~d}, 2 \mathrm{H}, J=8.2 \mathrm{~Hz}) ;{ }^{13} \mathrm{C}$ RMN (75 MHz, Acetone- $\left.d_{6}\right): \delta$ 144.0, 140.9, 139.7, 138.8, 137.8, 133.4, $131.7(\mathrm{c}, J=32.1 \mathrm{~Hz}), 126.5,126.4$, (c, $J=4.4 \mathrm{~Hz}), 125.8,125.7,124.2,122.8,121.7,99.0$, 84.4. MS $\left(\mathrm{ESI}^{+}\right) \mathrm{m} / \mathrm{z} 298\left(\mathrm{M}^{+}, 100\right)$. Anal. Calcd for $\mathrm{C}_{18} \mathrm{H}_{11} \mathrm{~F}_{9} \mathrm{NP}: \mathrm{C}, 48.78 ; \mathrm{H}, 2.50 ; \mathrm{N}, 3.16$. Found: C, 48.84; H, 2.73; N, 3.28 .

\section{4-(4-Methoxy-phenylethynyl)-quinolizinium hexafluorophosphate (4a).}

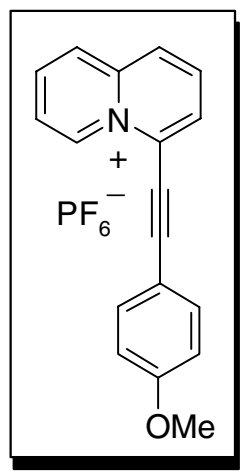

Following the general procedure A, from 5d and 1-ethynyl-4-methoxybenzene $(27 \mu \mathrm{l})$, stirring at room temperature for $14 \mathrm{~h}$, afforded $43.0 \mathrm{mg}$ (61\%) of $\mathbf{4 a}$ as a yellow solid: $\mathrm{mp} 220-222^{\circ} \mathrm{C}$; IR (KBr) $3119,2201,1601$, 1512, 1258, 839, $558 \mathrm{~cm}^{-1}$; ${ }^{1} \mathrm{H}$ RMN (300 MHz, Acetone- $\left.d_{6}\right): \delta 10.08$ (d, $1 \mathrm{H}, J=7.0 \mathrm{~Hz}), 8.77(\mathrm{~d}, 1 \mathrm{H}, J=8.4 \mathrm{~Hz}), 8.70-8.65(\mathrm{~m}, 1 \mathrm{H}), 8.58(\mathrm{ddd}, 1 \mathrm{H}$, $J=9.7,7.3,1.1 \mathrm{~Hz}), 8.53-8.47(\mathrm{~m}, 2 \mathrm{H}), 8.33(\mathrm{td}, 1 \mathrm{H}, J=7.0,1.5 \mathrm{~Hz}), 7.84$ 
$(\mathrm{d}, 2 \mathrm{H}, J=9.0 \mathrm{~Hz}), 7.13(\mathrm{~d}, 2 \mathrm{H}, J=9.0 \mathrm{~Hz}), 3.91(\mathrm{~s}, 3 \mathrm{H}) ;{ }^{13} \mathrm{C} \mathrm{RMN}\left(75 \mathrm{MHz}\right.$, Acetone- $\left.d_{6}\right): \delta$ 163.2, 144.7, 138.6, 136.9, 135.7, 135.3, 130.4, 129.4, 127.9, 126.0, 119.6, 115.7, 112.3, 107.7, 79.4, 56.1. MS $\left(\mathrm{ESI}^{+}\right) \mathrm{m} / \mathrm{z} 260\left(\mathrm{M}^{+}, 100\right)$. Anal. Calcd for $\mathrm{C}_{18} \mathrm{H}_{14} \mathrm{~F}_{6} \mathrm{NOP}: \mathrm{C}, 53.35 ; \mathrm{H}, 3.48 ; \mathrm{N}$, 3.46. Found: C, 53.60; H, 3.59; N, 3.27.

\section{4-(4-Trifluoromethyl-phenylethynyl)-quinolizinium hexafluorophosphate (4b).}

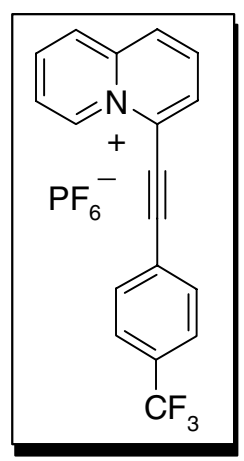

Following the general procedure A, from 5d and 1-ethynyl-4trifluoromethyl-benzene $(34 \mu \mathrm{l})$, tirring at room temperature for $14 \mathrm{~h}$, afforded $38.4 \mathrm{mg}(50 \%)$ de $\mathbf{4 b}$ as a white solid: $\mathrm{mp} 213-215^{\circ} \mathrm{C}$; IR $(\mathrm{KBr})$ $3121,2927,222,1641,1618,1411,1329,1129,1068,837,558 \mathrm{~cm}^{-1} ;{ }^{1} \mathrm{H}$ RMN (300 MHz, Acetone- $\left.d_{6}\right): \delta 10.16(\mathrm{dd}, 1 \mathrm{H}, J=7.0,0.9 \mathrm{~Hz}), 8.82(\mathrm{~d}$, $1 \mathrm{H}, J=8.6 \mathrm{~Hz}), 8.77(\mathrm{~d}, 1 \mathrm{H}, J=8.6 \mathrm{~Hz}), 8.66-8.61(\mathrm{~m}, 2 \mathrm{H}), 8.54(\mathrm{t}, 1 \mathrm{H}, J=$ $7.5 \mathrm{~Hz}), 8.36(\mathrm{td}, 1 \mathrm{H}, J=7.1,1.6 \mathrm{~Hz}), 8.13(\mathrm{~d}, 2 \mathrm{H}, J=8.1 \mathrm{~Hz}), 7.93(\mathrm{~d}, 2 \mathrm{H}$, $J=8.1 \mathrm{~Hz}) ;{ }^{13} \mathrm{C}$ RMN $\left(75 \mathrm{MHz}\right.$, Acetone- $\left.d_{6}\right): \delta 144.8,139.1,136.8,136.0$, 134.0, 132.7 (c, $J=32.3 \mathrm{~Hz}$ ), 130.6, 129.6, 129.3, 129.1, 126.7 (c, $J=3.7 \mathrm{~Hz}), 126.3,124.9$, $124.7(\mathrm{c}, J=267.5 \mathrm{~Hz}), 104.0,81.7 . \mathrm{MS}\left(\mathrm{ESI}^{+}\right) \mathrm{m} / \mathrm{z} 298\left(\mathrm{M}^{+}, 100\right)$. Anal. Calcd for $\mathrm{C}_{18} \mathrm{H}_{11} \mathrm{~F}_{9} \mathrm{NP}: \mathrm{C}, 48.78 ; \mathrm{H}, 2.50 ; \mathrm{N}, 3.16$. Found: $\mathrm{C}, 48.51 ; \mathrm{H}, 2.66 ; \mathrm{N}, 3.10$. 